\title{
Epigenetic aberrations in myeloid malignancies (Review)
}

\author{
SHINICHIRO TAKAHASHI
}

\begin{abstract}
Division of Hematology, Kitasato University School of Allied Health Sciences; Division of Molecular Hematology, Kitasato University Graduate School of Medical Sciences, Sagamihara, Kanagawa 252-0373, Japan
\end{abstract}

Received February 15, 2013; Accepted April 16, 2013

DOI: $10.3892 /$ ijmm.2013.1417

\begin{abstract}
The development of novel technologies, such as massively parallel DNA sequencing, has led to the identification of several novel recurrent gene mutations, such as DNA methyltransferase (Dnmt)3a, ten-eleven-translocation oncogene family member 2 (TET2), isocitrate dehydrogenase (IDH)1/2, additional sex comb-like 1 (ASXL1), enhancer of zeste homolog 2 (EZH2) and ubiquitously transcribed tetratricopeptide repeat X chromosome (UTX) mutations in acute myeloid leukemia (AML) and other myeloid malignancies. These findings strongly suggest a link between recurrent genetic alterations and aberrant epigenetic regulations, resulting from an abnormal DNA methylation and histone modification status. This review focuses on the current findings of aberrant epigenetic signatures by these newly described genetic alterations. Moreover, epigenetic aberrations resulting from transcription factor aberrations, such as mixed lineage leukemia (MLL) rearrangement, ecotropic viral integration site 1 (Evi1) overexpression, chromosomal translocations and the downregulation of PU.1 are also described.
\end{abstract}

\section{Contents}

1. Introduction

2. TET2 mutations

3. IDH1/2 mutations

4. Dnmt mutations

5. ASXL1 mutations

6. EZH2 mutations

7. UTX mutations

8. Epigenetic aberrations result from transcription factor aberrations - MLL rearrangement

9. Evil overexpression

10. Chromosomal translocations

Correspondence to: Professor Shinichiro Takahashi, Division of Hematology, Kitasato University School of Allied Health Sciences, 1-15-1 Kitasato, Minami-ku, Sagamihara, Kanagawa 252-0373, Japan E-mail: shin@kitasato-u.ac.jp

Key words: gene mutations, myeloid malignancies, epigenetic aberrations
11. PU.1 downregulation

12. Conclusion

\section{Introduction}

The development of novel technologies, such as massively parallel DNA sequencing, has led to the identification of several important genetic mutations in acute myeloid leukemia (AML) (1). These include ten-eleven-translocation oncogene family member 2 (TET2), isocitrate dehydrogenase (IDH)1 (2) and DNA methyltransferase (Dnmt)3a mutations (3). These mutations are frequently found in cytogenetically normal AML (1). In particular, TET2 and IDH1/2 mutations lead to the aberrant hypermethylation signature in AML cells (4). In addition, there are specific alterations in the gene expression profiles, such as homeobox $(H o x) B$ genes, in samples with Dnmt3a mutations compared with those without such mutations (5). These findings strongly suggest a link between recurrent genetic alterations and aberrant epigenetic regulation, resulting in an abnormal DNA methylation status in myeloid malignancies. This review focuses on the current findings of aberrant epigenetic signatures by these newly described genetic alterations. Moreover, epigenetic aberrations resulting from transcription factor aberrations are also described.

\section{TET2 mutations}

In 2009, Delhommeau et al (6) conducted a combination of molecular, cytogenetic, comparative genomic hybridization, and single nucleotide polymorphism analyses to identify a candidate tumor suppressor genes common in patients with myelodysplastic syndromes (MDS), myeloproliferative disorders and AML. They identified inactivating mutations of the TET2 gene in approximately $15 \%$ of patients with various myeloid malignancies, such as MDS (19\%), myeloproliferative disorders (12\%), secondary AML (24\%) and chronic myelomonocytic leukemia (CMML) (22\%) (6). In a recent study, Metzeler et al (7) analyzed 427 patients with cytogenetically normal (CN)-AML, and revealed TET2 mutations in $23 \%$ of the patients, associated with older age $(\mathrm{p}<0.001)$ and higher pretreatment white blood cell (WBC) $(\mathrm{p}=0.04)$ counts compared with wild-type TET2. A subsequent analysis of TET2 sequence and rearrangements in more than 1,000 patients by several groups led to the establishment of the frequencies of TET2 defects in myeloproliferative neoplasms (MPNs): approxi- 
mately $12 \%$ in all MPNs, $14 \%$ in polycythemia vera, $8 \%$ in essential thrombocythemia, $20 \%$ in primary myelofibrosis and $25 \%$ in post-MPN AML (8).

TET2 can convert 5-methylcytosine $(5-\mathrm{mC})$ to 5-hydroxymethylcytosine (5-hmC) (9), which was to be an intermediate in the demethylation of DNA. Bone marrow samples from patients with TET2 mutations displayed uniformly low levels of 5-hmC in genomic DNA compared to bone marrow samples from healthy controls (9). Figueroa et al (4) investigated TET2 mutant AMLs and identified a hypermethylation phenotype, including 129 differentially methylated regions. However, the precise molecular mechanism of TET-mediated gene regulation has not yet been elucidated. Recently, Chen et al (10), using protein affinity purification, demonstrated that TET 2 and TET3 are associated with $O$-linked $\beta$-N-acetylglucosamine $(O$-GlcNAc) transferase (OGT), an enzyme that on its own catalyzes the addition of $O$-GlcNAc onto serine (Ser) and threonine residues $(O$-GlcNAcylation) in vivo. TET2 directly interacts with OGT, and recruits OGT to chromatin. The authors revealed that the OGT-mediated $O$-GlcNAcylation of Ser 122 of histone H2B contributes to TET2-dependent gene transcriptional activation.

Recurrent mutations of the TET2 gene in myeloid malignancies may disrupt these epigenetic machineries, leading to aberrant gene regulation, and may play a role in the pathogenesis of these diseases.

\section{IDH1/2 mutations}

Mardis et al (2), using massively parallel DNA sequencing to obtain a very high level of coverage of a primary, cytogenetically normal, de novo genome for AML with minimal maturation (AML-M1) and a matched normal skin genome, identified 12 acquired mutations within the coding sequences of genes and 52 somatic point mutations in conserved or regulatory portions of the genome. Many of them were mutations that have already been identified, such as NRAS and nucleophosmin (NPM)1, but they found a novel mutaton of the IDHI gene (2). They found that the IDHI gene mutation was present in 15 out of the 187 AML genomes and was strongly associated with a CN status. Boissel et al (11) analyzed both IDH1 mutations and IDH 2 mutations in a cohort of 520 adults with AML homogeneously treated in the French Acute Leukemia French Association (ALFA) 9801 and 9802 trials. The prevalence of IDH1 and IDH2 mutations was 9.6 and $3.0 \%$, respectively, mostly associated with a CN status. Schnittger et al (12) conducted larger study. They analyzed IDH1R132 mutations (Fig. 1) in 1,414 AML patients and detected IDH1 mutations in $6.6 \%$ of 1,414 AML patients with a clear prevalence in the intermediate risk karyotype group $(10.4 \%, \mathrm{p}<0.001)$. They also detected IDH1 mutations with strong associations not only with NPM1 mutations $(\mathrm{p}<0.001)$ but also with partial tandem duplications (PTDs) in the mixed lineage leukemia (MLL) gene $(\mathrm{p}=0.020)$.

IDH1/2 function at a crossroads of cellular metabolism in lipid synthesis, cellular defense against oxidative stress, oxidative respiration and oxygen sensing signal transduction (13). Recently, it was demonstrated that AML patients harboring IDH1 and 2 mutations displayed aberrant hypermethylation (4). In a recent study by Duncan et al (14), the authors used a gene-targeting approach by the addition of 'knocking-in' a single copy of the most frequently observed IDH1 mutation, $\mathrm{R} 132 \mathrm{H}$, into a human cancer cell line and profiled changes in DNA methylation at over $27,000 \mathrm{CpG}$ dinucleotides relative to wild-type parental cells. They found that IDH1(R132H/WT) mutation induces widespread alterations in DNA methylation, including hypermethylation of 2010 and hypomethylation of $842 \mathrm{CpG}$ loci. Furthermore, they showed that the direction of IDH1(R132H/WT)-mediated DNA methylation change was largely dependent upon pre-existing DNA methylation levels, resulting in the depletion of moderately methylated loci (14).

Consistent with another study (7), IDH1/2 mutations were shown to be mutually exclusive with mutations in TET2 $(p=0.009)$ (4). In fact, these mutations led to the production of an abnormal metabolite in the cell, 2-hydroxyglutarate, which inhibits the hydroxylation of 5-mC by TET2 (4). In agreement with these results, Metzeler et al (7) also demonstrated that IDH1/2 mutations were less frequent in TET2-mutated than in TET2 wild-type patients $(\mathrm{p}<0.001)$, suggesting that these mutations are mutually exclusive.

\section{Dnmt mutations}

Dnmts are enzymes that catalyze the addition of methyl groups to the cytosine residue of $\mathrm{CpG}$ dinucleotides. Aberrant $\mathrm{CpG}$ island methylation has long been hypothesized to contribute to the pathogenesis of cancer (15). Using massively parallel DNA sequencing (16), Ley et al (3) identified a somatic mutation in Dnmt3a, sequencing from 116.4 billion base pairs of sequence and $99.6 \%$ diploid coverage of the genome of cells from a patient with AML with a normal karyotype. Further analysis revealed Dnmt3a mutations in 62 of 281 AML patients (22.1\%). The structure of Dnmt3a, and the region where mutations frequently occur, is presented in Fig. 1. Yan et al (5) revealed that although Dnmt3a mutations do not dramatically alter global DNA methylation levels in AML genomes, there were alterations of specific gene DNA methylation patterns and/ or gene expression profiles, such as HoxB genes, in samples with Dnmt3a mutations as compared with those without such changes (5). Consistent with these data, the Dnmt3a mutation, which frequently occurs in arginine (R)882, showed reduced enzymatic activity in vitro (5).

\section{ASXL1 mutations}

The additional sex comb-like $1(A S X L 1)$ gene belongs to a family of three identified members that encode proteins regulating chromatin remodeling. The ASXL proteins contain a $\mathrm{C}$-terminal plant homeodomain (PHD) finger and belong to the polycomb and trithorax complexes, which regulate the genetic program of stem cells. ASXL1 is involved in the regulation of histone methylation through the cooperation with heterochromatin protein-1 to modulate the activity of lysine-specific demethylase 1 (17), a histone demethylase for $\mathrm{K} 4$ of histone $\mathrm{H} 3, \mathrm{~K} 9$ of histone $\mathrm{H} 3$ and also important for global DNA methylation (18). Recent studies have identified mutations of the ASXL1 gene in various types of myeloid malignancies, including approximately $10 \%$ of MDS (19), $10 \%$ of MPNs (20) and 40\% of CMML cases (19). Recently, Chou et al (21) examined ASXL1 mutations in exon 12 in 501 adults with de novo AML. ASXL1 mutations 

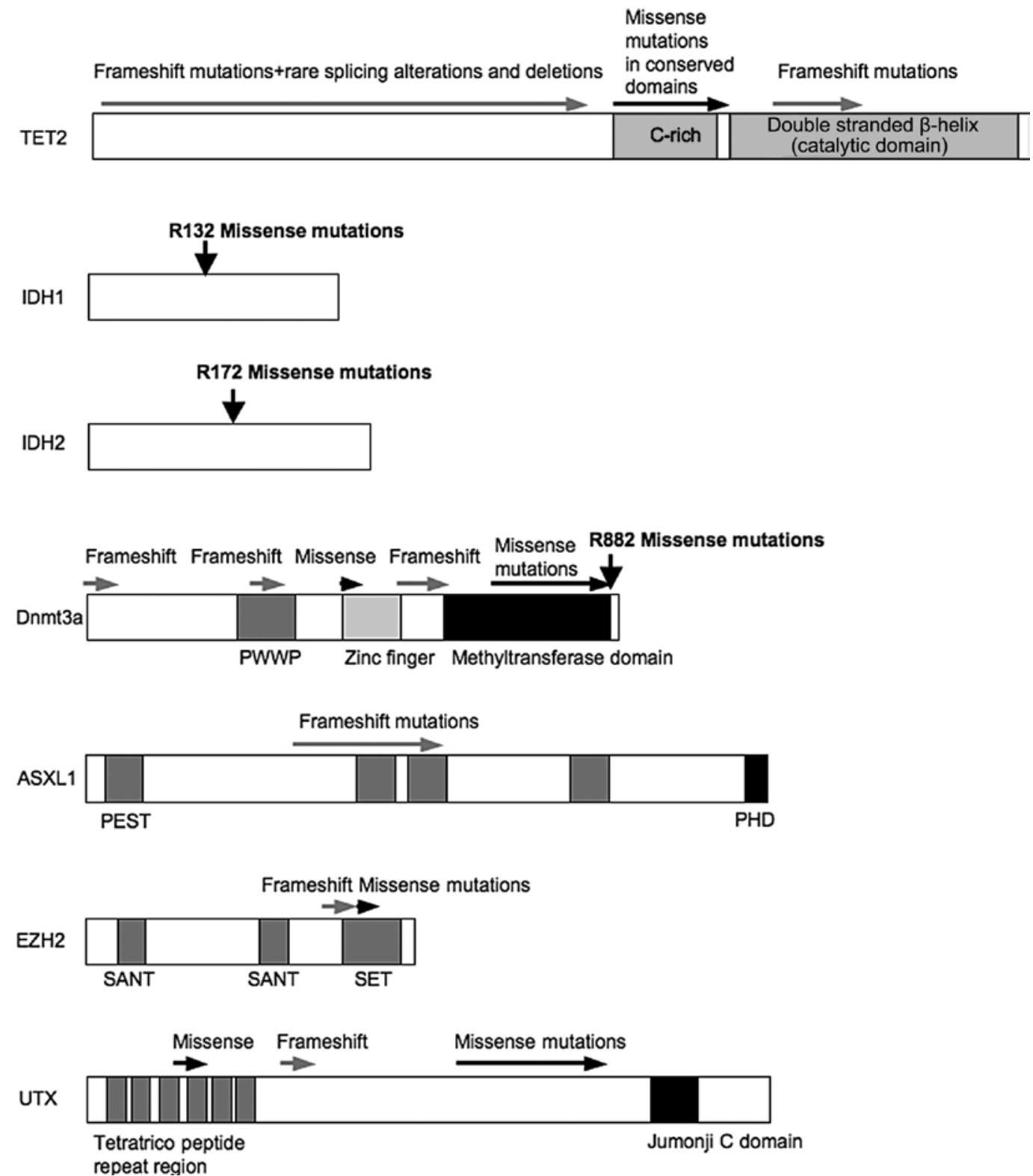

Figure 1. Mutations of factors play a role in epigenetic gene regulations in myeloid malignancies. Common mutations, shown in isocitrate dehydrogenase (IDH) 1, IDH2 and DNA methyltransferase (Dnmt) 3a are shown in vertical arrows with bold letters. Frameshift mutations are indicated by gray horizontal arrows and missense mutations are indicated by black horizontal arrows. These mutations are the data from myeloproliferative neoplasms (MPNs) (polycythemia vera, essential thrombocythemia, primary myelofibrosis) for ten-eleven-translocation oncogene family member 2 (TET2) (8). acute myeloid leukemia (AML) for Dnmt3a (3), myelodysplastic syndromes (MDS) for additional sex comb-like 1 (ASXL1) (8), enhancer of zeste homolog 2 (EZH2) (29) and ubiquitously transcribed tetratricopeptide repeat X chromosome (UTX) (29).

were detected in 54 patients (10.8\%), 8.9\% among those with the normal karyotype and $12.9 \%$ among those with abnormal cytogenetics. The mutation was closely associated with older age, the male gender, isolated trisomy 8 , runt-related transcription factor 1 (RUNX1) mutation, the expression of human leukocyte antigen-DR and CD34, but was inversely associated with $\mathrm{t}(15 ; 17)$, complex cytogenetics, internal tandem duplication of Fms-like tyrosine kinase 3, NPM1 mutations, Wilms tumor 1 mutations and the expression of CD33 and CD15. Patients with ASXL1 mutations had a shorter overall survival than patients without these mutations, but the mutation was not an independent adverse prognostic factor in multivariate analysis (21). However, hematopoietic fuction of ASXL1 remains unclear, since the study of ASXL1 knockout mice revealed only the mild hematopoietic phenotype (22).

\section{EZH2 mutations}

Enhancer of zeste homolog 2 (EZH2) is located at 7q36.1 and functions as the catalytic domain of the polycomb repressive complex 2 (PRC2), causing gene silencing by the trimethylation of K27 of histone H3. Abnormalities of chromosome 7q are common in myeloid malignancies (23). Recently, several studies have identified a minimally affected region on $7 \mathrm{q}$ that ultimately led to the discovery of mutations in EZH2 in several myeloid malignancies (24-26). In myeloid neoplasms, mutations have been described largely in poor prognosis myelodysplasia-MPNs (10-13\%), myelofibrosis (13\%), and various subtypes of MDS $(24,25,27,28)$. EZH2 mutations are frequently associated with uniparental disomy $7 \mathrm{q}$ or $7 \mathrm{q} 36.1$ microdeletion, but not monosomy 7 and $\operatorname{del}(7 \mathrm{q})$. EZH2 
mutations in myeloid neoplasms were spread throughout the gene and comprised of frameshift, missense and nonsense mutations (Fig. 1), some of which were homozygous. Since the catalytic SET domain lies at the far C-terminus, all nonsense and stop codon mutations would be predicted to result in the loss of histone methyltransferase activity (27). By contrast, the mutation of a single residue (Y641) and gain of function is observed in lymphoma (27). The fact that both activating and inactivating mutations of EZH2 are associated with malignancy reflects the complex role that polycomb group genes play in cell fate decisions (29).

\section{UTX mutations}

Ubiquitously transcribed tetratricopeptide repeat, X chromosome (UTX) may also play an important role in epigenetic regulation. It is a histone $\mathrm{H} 3 \mathrm{~K} 27$ demethylase and belongs to the polycomb group of proteins. van Haaften et al (30) demonstrated the inactivating somatic mutations in the UTX, pointing to histone $\mathrm{H} 3$ lysine methylation deregulation in multiple tumor types. They also revealed that UTX reintroduction into cancer cells with inactivating UTX mutations resulted in the suppression of proliferation and marked transcriptional changes (30). Of note, in cells transfected with wild-type UTX, SRY-related HMG-box 21 and protocadherin 19, two genes showing significant expression changes in the transfected UTX null lines, demonstrated a significant decrease in the trimethylation of histone H3K27 levels upon UTX reintroduction, suggesting that the expression changes were a direct effect of reconstituted UTX demethylase activity (30). These data indicate that the mutational inactivation of UTX occurs, at least in part, through transcriptional control mechanisms.

Gelsi-Boyer et al (19) reported rare deletions of UTX and other polycomb group protein family members in MDS. Jankowska et al (28) recently revealed that in a cohort of 73 patients with CMML and AML-derived CMML, two somatic, inactivating UTX mutations were detected and four additional missense variants that could correspond to rare polymorphisms were identified.

\section{Epigenetic aberrations result from transcription factor aberrations - MLL rearrangement}

Approximately $4-11 \%$ of patients with CN-AML present with rearrangement of the $M L L$ gene as the result of a PTD within a single MLL allele (31). MLL is a $430-\mathrm{kDa}$ transcription factor and has a complex structure that includes three AT-hook domains for DNA binding, a methyltransferase homology domain and a SET domain (31). MLL is necessary for the maintenance of Hox gene expression (32). The MLL SET domain can bind the promoter of Hox genes (33). Dorrance et al (34) generated a mouse knock-in model in which exons 5 through 11 of the murine MLL gene were targeted to intron 4 of the endogenous $M l l$ locus. $M l l^{\mathrm{PTD} / \mathrm{WT}}$ mice exhibit an alteration in the boundaries of normal Hox gene expression during embryogenesis, resulting in axial skeletal defects and increased numbers of hematopoietic progenitor cells. $M l l^{\mathrm{PTD} /}$ ${ }^{\text {wT }}$ mice overexpress Hoxa7, Hoxa9 and Hoxa10 in the spleen, bone marrow (BM) and blood. An increase in histone $\mathrm{H} 3 / \mathrm{H} 4$ acetylation and histone $\mathrm{H} 3 \mathrm{~K} 4$ methylation within the Hoxa7 and Hoxa9 promoters provides an epigenetic mechanism by which this overexpression occurs in vivo and an etiological role for MLL-PTD gain-of-function in the pathogenesis in AML. Consistent with this, Whitman et al (35) reported that AML patients with MLL-PTD exhibited increased global DNA methylation vs. AML patients with MLL-wild-type. Munoz et al (36) examined 93 adult patients with de novo AML for incidence clinical features of MLL rearranged AMLs. As a result, they revealed that MLL rearrangements were detected in $13(14 \%)$ patients. In the French-American-British Classification, there was a significantly higher percentage of M5 sybtypes in the MLL rearranged group. MLL rearranged patients had a lower event-free survival (EFS) $(\mathrm{p}=0.001)$ and a higher probability of relapse (REL) compared to MLL wild-type patients $(\mathrm{p}=0.07)$.

\section{Evil overexpression}

The abnormal expression of the ecotropic viral integration site 1 (Evil) gene, as the result of $\operatorname{inv}(3)(\mathrm{q} 21 \mathrm{q} 26) / \mathrm{t}(3,3)(\mathrm{q} 21 ; \mathrm{q} 26.2)$ or through other unknown mechanisms, is associated with unfavorable AML outcome (37,38). Evil encodes a $\mathrm{C} 2 \mathrm{H} 2$ zinc finger transcription factor, binding DNA in a sequencespecific manner and functions as a repressor (39-41). Lugthart et al (42) examined the aberrant epigenetic programming in AML patients, conducting a large-scale DNA methylation profiling study in human Evil AMLs. They compared and contrasted the abundance of cytosine methylation at 14,000 gene promoters using the HpaII tiny fragment enrichment by ligation-mediated PCR (HELP) assay in 26 AML patients overexpressing Evil and in eight $\mathrm{CD} 34^{+}$normal bone marrow controls. The signature contained 294 differentially methylated genes, of which 238 (81\%) were coordinately hypermethylated. These data suggest that Evil contributes to aberrant promoter DNA methylation patterning in leukemia.

\section{Chromosomal translocations}

From the biological standpoint, genome AML-associated fusion proteins that result from chromosomal translocations have been reported to aid in the establishment of specific DNA methylation patterns in AML. For example, promyelocytic leukemia (PML)/retinoic acid receptor (RAR) $\alpha$ and RUNX1/eight-twenty one oncoprotein (ETO) have been to used recruit both histone deacetylases and DNA methyltransferases to induce the transcriptional repression of target genes (43).

Figueroa et al (44) recently examined the methylation profiles of 344 patients with AML. They demonstrated that established AML1-ETO $(n=24,7 \%)$, core binding factor $\beta$ (CBF $\beta$ )-myosin, heavy chain 11, smooth muscle (MYH11) $(n=30,9 \%)$ and PML-RAR $\alpha(n=10,3 \%)$ leukemia entities were associated with specific methylation profiles. In addition, they revealed that DNA methylation profiles segregated patients with CCAAT/enhancer-binding protein (CEBP) $\alpha$ aberrations from other subtypes of leukemia. They also defined epigenetically distinct forms of AML with NPM1 mutations. Although the molecular mechanisms of these genetic alterations and specific methylation remain unsolved, it is likely that the disruption of transcriptional repressor function by chromosomal translocation may contribute to the aberrant hypermethylation profile in AML cells. 
Table I. A list of genetic aberrations with the phenotype associated with epigenetic aberrations.

Genetic aberrations

Epigenetic aberrations

Refs.

TET2 mutations

TET2 can convert 5-methylcytosine (5-mC) to 5-hydroxymethylcytosine (5-hmC),

which was to be an intermediate in the demethylation of DNA. Uniformly low levels of 5-hmC in genomic DNA compared to bone marrow samples from healthy controls.

TET2 directly interacts with $O$-linked $\beta$-N-acetylglucosamine ( $O$-GlcNAc) transferase (OGT), and recruits OGT to chromatin. OGT-mediated O-GlcNAcylation of Ser 122 of histone H2B contributes to TET2-dependent gene expression.

IDH1/2 mutations Global hypermethylation.

Dnmt3a mutations Although Dnmt3a mutations do not dramatically alter global DNA methylation levels in

AML genomes, there were alterations of $H o x B$ gene DNA methylation patterns

and gene expression profiles in samples with Dnmt3a mutations

as compared with those without such changes.

ASXL1 mutations Unclear. The study of ASXL1 knockout mice revealed only the mild hematopoietic phenotype.

EZH2 mutations

Unclear. All nonsense and stop codon mutations would be predicted to result in the loss of

UTX mutations Inactivating somatic mutations in UTX, pointing to histone H3 lysine methylation

deregulation in multiple tumor types. UTX reintroduction into cancer cells with

inactivating UTX mutations resulted in the suppression of proliferation

and marked transcriptional changes.

MLL-PTD From the analysis of MLL-PTD knock-in mice, an increase in histone H3/H4 acetylation and histone H3 lysine 4 (Lys4) methylation within the Hoxa7 and Hoxa9 promoters was observed.

Evi1 overexpression Analysis from a large-scale DNA methylation profiling study in human EVI1 AMLs revealed that the signature contained 294 differentially methylated genes, of which $238(81 \%)$ were coordinately hypermethylated.

AML1-ETO, AML1-ETO, CBFß-MYH11 and PML-RAR $\alpha$ leukemia entities are associated with CBFß-MYH11, specific methylation profiles. PML-RAR $\alpha$

Downregulation of Metallothionein-1, vimentin genes were upregulated, with increases in acetylated histone H3 PU.1 and $\mathrm{H} 4$ levels, and decreases in DNA methylation in their promoters.

TET2, ten-eleven-translocation oncogene family member 2; IDH1/2, isocitrate dehydrogenase 1/2; Dnmt3a, DNA methyltransferase 3a; AML, acute myeloid leukemia; HoxB, homeobox B; ASXL1, additional sex comb-like 1; EZH2, enhancer of zeste homolog 2; UTX, ubiquitously transcribed tetratricopeptide repeat X chromosome; MLL, mixed lineage leukemia; PTD, partial tandem duplication; Evi1, ecotropic viral integration site 1; ETO, eight-twenty one oncoprotein; CBF $\beta$, core binding facgtor $\beta$; MYH11, myosin, heavy chain 11 , smooth muscle; PML, promyelocytic leukemia; RAR $\alpha$, retinoic acid receptor $\alpha$.

\section{PU.1 downregulation}

The overall incidence of the genetic aberration of the PU.I gene is extremely rare in myeloid leukemia (45-47). However, the level of PU.1 expression is critical for specifying the fate of cells and, if perturbed, even modest decreases in PU.1 levels can lead to the development of leukemia and lymphoma $(48,49)$. Indeed, PU.1 is expressed at low levels in the majority of cases of human AML (50). Similar to Evil (42), PU.1 interacts with Dnmt3a and Dnmt3b (51) and suppresses its target genes through Dnmt activity $(51,52)$. In addition, it was recently revealed that changes in PU.1 expression also affect several genes (metallothionein-1, vimentin), playing a role in cellular proliferation and differentiation, promoter DNA methylation and histone acetylation levels in leukemia cells $(52,53)$. Dnmt3a mutation is prevalent in AML, and the expression of Dnmt3s is constant in AML specimens (3). Therefore, it is possible that Dnmt3a mutations exert their leukemogenic potential, at least in part, through the functional impairment of PU.1 and/or Evi1 epigenetic activity as a transcriptional repressor, mainly through the downregulation of its expression. Further studies are required in order to fully elucidate the mechanisms involved.

\section{Conclusion}

The development of novel technologies has led us to detect several important genetic mutations in AML. Along with the development of whole genome sequencing, major genetic aberrations have been almost completely identified. The next 
stage is to determine the consequence of these molecular alterations, particularly for these newly identified molecules. A list of genetic aberrations with the phenotype associated with epigenetic aberrations is summarized in Table I.

These newly identified combinations of genetic aberrations may lead to a refined disease classification and to the development of rational, epigenetic or signal transduction pathway targeted therapies.

\section{Acknowledgements}

The present study was supported in part by Grants-in-Aid for Scientific Research (no. 23590687) from the Ministry of Education, Science and Culture, Japan, and the Takeda Science Foundation, a foundation from Kitasato University School of Allied Health Sciences (Grant-in-Aid for Research Project, no. 2012-1002).

\section{References}

1. Takahashi S: Current findings for recurring mutations in acute myeloid leukemia. J Hematol Oncol 4: 36, 2011.

2. Mardis ER, Ding L, Dooling DJ, et al: Recurring mutations found by sequencing an acute myeloid leukemia genome. N Engl J Med 361: 1058-1066, 2009.

3. Ley TJ, Ding L, Walter MJ, et al: DNMT3A mutations in acute myeloid leukemia. N Engl J Med 363: 2424-2433, 2010

4. Figueroa ME, Abdel-Wahab O, Lu C, et al: Leukemic IDH1 and IDH2 mutations result in a hypermethylation phenotype, disrupt TET2 function, and impair hematopoietic differentiation. Cancer Cell 18: 553-567, 2010.

5. Yan XJ, Xu J, Gu ZH, et al: Exome sequencing identifies somatic mutations of DNA methyltransferase gene DNMT3A in acute monocytic leukemia. Nat Genet 43: 309-315, 2011.

6. Delhommeau F, Dupont S, Della Valle V, et al: Mutation in TET2 in myeloid cancers. N Engl J Med 360: 2289-2301, 2009.

7. Metzeler KH,Maharry K, Radmacher MD, et al: TET2 mutations improve the new European LeukemiaNet risk classification of acute myeloid leukemia: a Cancer and Leukemia Group B study. J Clin Oncol 29: 1373-1381, 2011.

8. Delhommeau F, Jeziorowska D, Marzac C and Casadevall N: Molecular aspects of myeloproliferative neoplasms. Int J Hematol 91: 165-173, 2010.

9. Ko M, Huang Y, Jankowska AM, et al: Impaired hydroxylation of 5-methylcytosine in myeloid cancers with mutant TET2. Nature 468: 839-843, 2010.

10. Chen Q, Chen Y, Bian C, Fujiki R and Yu X: TET2 promotes histone $O$-GlcNAcylation during gene transcription. Nature 493: 561-564, 2013.

11. Boissel N, Nibourel O, Renneville A, et al: Prognostic impact of isocitrate dehydrogenase enzyme isoforms 1 and 2 mutations in acute myeloid leukemia: a study by the Acute Leukemia French Association group. J Clin Oncol 28: 3717-3723, 2010.

12. Schnittger S, Haferlach C, Ulke M, Alpermann T, Kern W and Haferlach T: IDH1 mutations are detected in 6.6\% of 1414 AML patients and are associated with intermediate risk karyotype and unfavorable prognosis in adults younger than 60 years and unmutated NPM1 status. Blood 116: 5486-5496, 2010

13. Reitman ZJ and Yan H: Isocitrate dehydrogenase 1 and 2 mutations in cancer: alterations at a crossroads of cellular metabolism. J Natl Cancer Inst 102: 932-941, 2010.

14. Duncan CG, Barwick BG, Jin G, et al: A heterozygous IDH1R132H/WT mutation induces genome-wide alterations in DNA methylation. Genome Res 22: 2339-2355, 2012.

15. Esteller M: Epigenetics in cancer. N Engl J Med 358: 1148-1159, 2008.

16. Wheeler DA, Srinivasan M, Egholm M, et al: The complete genome of an individual by massively parallel DNA sequencing. Nature 452: 872-876, 2008.

17. Lee SW, Cho YS, Na JM, et al: ASXL1 represses retinoic acid receptor-mediated transcription through associating with HP1 and LSD1. J Biol Chem 285: 18-29, 2010.
18. Wang J, Hevi S, Kurash JK, et al: The lysine demethylase LSD1 (KDM1) is required for maintenance of global DNA methylation. Nat Genet 41: 125-129, 2009.

19. Gelsi-Boyer V, Trouplin V, Adelaide J, et al: Mutations of polycomb-associated gene ASXL1 in myelodysplastic syndromes and chronic myelomonocytic leukaemia. Br J Haematol 145: 788-800, 2009.

20. Abdel-Wahab O, Manshouri T, Patel J, et al: Genetic analysis of transforming events that convert chronic myeloproliferative neoplasms to leukemias. Cancer Res 70: 447-452, 2010.

21. Chou WC, Huang HH, Hou HA, et al: Distinct clinical and biological features of de novo acute myeloid leukemia with additional sex comb-like 1 (ASXL1) mutations. Blood 116: 4086-4094, 2010.

22. Fisher CL, Pineault N, Brookes C, et al: Loss-of-function additional sex combs like 1 mutations disrupt hematopoiesis but do not cause severe myelodysplasia or leukemia. Blood 115: 38-46, 2010.

23. Tiu RV, Visconte V, Traina F, Schwandt A and Maciejewski JP: Updates in cytogenetics and molecular markers in MDS. Curr Hematol Malig Rep 6: 126-135, 2011.

24. Nikoloski G, Langemeijer SM, Kuiper RP, et al: Somatic mutations of the histone methyltransferase gene EZH2 in myelodysplastic syndromes. Nat Genet 42: 665-667, 2010.

25. Ernst T, Chase AJ, Score J, et al: Inactivating mutations of the histone methyltransferase gene EZH2 in myeloid disorders. Nat Genet 42: 722-726, 2010.

26. Makishima H, Jankowska AM, Tiu RV, et al: Novel homo- and hemizygous mutations in EZH2 in myeloid malignancies. Leukemia 24: 1799-1804, 2010.

27. Chase A and Cross NC: Aberrations of EZH2 in cancer. Clin Cancer Res 17: 2613-2618, 2011.

28. Jankowska AM, Makishima H, Tiu RV, et al: Mutational spectrum analysis of chronic myelomonocytic leukemia includes genes associated with epigenetic regulation: UTX, EZH2, and DNMT3A. Blood 118: 3932-3941, 2011.

29. Sauvageau M and Sauvageau G: Polycomb group proteins: multifaceted regulators of somatic stem cells and cancer. Cell Stem Cell 7: 299-313, 2010

30. van Haaften G, Dalgliesh GL, Davies H, et al: Somatic mutations of the histone H3K27 demethylase gene UTX in human cancer. Nat Genet 41: 521-523, 2009.

31. Basecke J, Whelan JT, Griesinger F and Bertrand FE: The MLL partial tandem duplication in acute myeloid leukaemia. Br J Haematol 135: 438-449, 2006.

32. Yu BD, Hess JL, Horning SE, Brown GA and Korsmeyer SJ: Altered Hox expression and segmental identity in Mll-mutant mice. Nature 378: 505-508, 1995.

33. Milne TA, Briggs SD, Brock HW, et al: MLL targets SET domain methyltransferase activity to Hox gene promoters. Mol Cell 10: 1107-1117, 2002.

34. Dorrance AM, Liu S, Yuan W, et al: Mll partial tandem duplication induces aberrant Hox expression in vivo via specific epigenetic alterations. J Clin Invest 116: 2707-2716, 2006.

35. Whitman SP, Hackanson B, Liyanarachchi S, et al: DNA hypermethylation and epigenetic silencing of the tumor suppressor gene, SLC5A8, in acute myeloid leukemia with the MLL partial tandem duplication. Blood 112: 2013-2016, 2008.

36. Munoz L, Nomdedeu JF, Villamor N, et al: Acute myeloid leukemia with MLL rearrangements: clinicobiological features, prognostic impact and value of flow cytometry in the detection of residual leukemic cells. Leukemia 17: 76-82, 2003.

37. Lugthart S, van Drunen E, van Norden Y, et al: High EVI1 levels predict adverse outcome in acute myeloid leukemia: prevalence of EVI1 overexpression and chromosome 3q26 abnormalities underestimated. Blood 111: 4329-4337, 2008.

38. Groschel S, Lugthart S, Schlenk RF, et al: High EVI1 expression predicts outcome in younger adult patients with acute myeloid leukemia and is associated with distinct cytogenetic abnormalities. J Clin Oncol 28: 2101-2107,

39. Delwel R, Funabiki T, Kreider BL, Morishita K and Ihle JN: Four of the seven zinc fingers of the Evi-1 myeloid-transforming gene are required for sequence-specific binding to $\mathrm{GA}(\mathrm{C} / \mathrm{T})$ AAGA(T/C)AAGATAA. Mol Cell Biol 13: 4291-4300, 1993.

40. Matsugi T, Kreider BL, Delwel R, Cleveland JL, Askew DS and Ihle JN: The Evi-1 zinc finger myeloid transforming protein binds to genomic fragments containing (GATA)n sequences. Oncogene 11: 191-198, 1995.

41. Perkins AS, Fishel R, Jenkins NA and Copeland NG: Evi-1, a murine zinc finger proto-oncogene, encodes a sequence-specific DNA-binding protein. Mol Cell Biol 11: 2665-2674, 1991. 
42. Lugthart S, Figueroa ME, Bindels E, et al: Aberrant DNA hypermethylation signature in acute myeloid leukemia directed by EVI1. Blood 117: 234-241, 2011.

43. Boultwood J and Wainscoat JS: Gene silencing by DNA methylation in haematological malignancies. Br J Haematol 138: 3-11, 2007.

44. Figueroa ME, Lugthart S, Li Y, et al: DNA methylation signatures identify biologically distinct subtypes in acute myeloid leukemia. Cancer Cell 17: 13-27, 2010.

45. Mueller BU, Pabst T, Osato M, et al: Heterozygous PU.1 mutations are associated with acute myeloid leukemia. Blood 100: 998-1007, 2002.

46. Vegesna V, Takeuchi S, Hofmann WK, et al: C/EBP-beta, C/EBP-delta, PU.1, AML1 genes: mutational analysis in 381 samples of hematopoietic and solid malignancies. Leuk Res 26: 451-457, 2002.

47. Lamandin C, Sagot C, Roumier C, et al: Are PU.1 mutations frequent genetic events in acute myeloid leukemia (AML)? Blood 100: 4680-4681, 2002.

48. DeKoter RP and Singh H: Regulation of B lymphocyte and macrophage development by graded expression of PU.1. Science 288: 1439-1441, 2000.
49. Rosenbauer F, Wagner K, Kutok JL, et al: Acute myeloid leukemia induced by graded reduction of a lineage-specific transcription factor, PU.1. Nat Genet 36: 624-630, 2004.

50. Steidl U, Rosenbauer F, Verhaak RG, et al: Essential role of Jun family transcription factors in PU.1 knockdown-induced leukemic stem cells. Nat Genet 38: 1269-1277, 2006.

51. Suzuki M, Yamada T, Kihara-Negishi F, et al: Site-specific DNA methylation by a complex of PU.1 and Dnmt3a/b. Oncogene 25: 2477-2488, 2006.

52. Imoto A, Okada M, Okazaki T, Kitasato H, Harigae $\mathrm{H}$ and Takahashi S: Metallothionein-1 isoforms and vimentin are direct PU.1 downstream target genes in leukemia cells. J Biol Chem 285: 10300-10309, 2010.

53. Suzuki S, Nakano H and Takahashi S: Epigenetic regulation of the metallothionein-1A promoter by PU.1 during differentiation of THP-1 cells. Biochem Biophys Res Commun 433: 349-353, 2013. 